\title{
Infarto agudo al miocardio en el adulto mayor. Características clínicas, evolución hospitalaria y a 5 años plazo
}

\author{
HÉCTOR UGALDE, DIEGO UGALDE, MACARENA MUÑOZ
}

Departamento de Cardiología. Hospital Clínico Universidad de Chile.

Recibido el 2 de septiembre de 2012, aceptado el 16 de octubre de 2013

Correspondencia a: Dr. Héctor Ugalde Departamento de Cardiología Hospital Clínico Universidad de Chile.

Av. Santos Dumont 999, Santiago-Chile. E-mail: ugaldehector@ gmail.com

\section{Features of acute myocardial infarction among aged patients}

Background: Among aged patients, acute myocardial infarction has more complications and there is a tendency to underuse proven treatments. Aim: To report the features of acute myocardial infarction among aged patients. Material and Methods: Analysis of a registry of patients with acute myocardial infarction admitted to a coronary unit. For the purposes of analysis, patients aged 65 years or more were selected. Follow up was made consulting medical records, calling patients by telephone or consulting death records at the National Identification Service. Results: A total of 1.358 patients were admitted in the study period with a diagnosis of acute myocardial infarction and 580 (43\%) were aged 65 years or more. On admission, this age group had a higher frequency of hemodynamic instability and anterior wall infarctions. Reperfusion therapy and beta blockers were used less commonly in this group. Hospital mortality among patients younger or older than 65 year was 8 and 25\%, respectively $(p<0.01)$. Among aged patients, five years mortality was 33\%. Conclusions: Among aged patients, acute myocardial infarction has a higher risk profile on admission, is usually undertreated and has higher mortality than younger subjects.

(Rev Med Chile 2013; 141: 1402-1410)

Key words: Aged; Mortality; Myocardial infarction.
E infarto agudo al miocardio (IAM) es la principal causa de muerte en nuestro país ${ }^{1,2}$ y la edad uno de los principales factores asociados a la mortalidad, lo que conduce a que la mortalidad en pacientes adultos mayores $(\geq$ 65 años) sea aun mayor ${ }^{1,2}$. En Estados Unidos de Norteamérica en el año 2002, 35\% de todas las muertes ocurría por esta causa en este grupo etario $^{3}$. Pese a su importancia como causa de muerte y discapacidad, existen pocas publicaciones en la literatura local y en el mundo sobre la caracterización, tratamiento y evolución del IAM en este subgrupo de pacientes, en particular en pacientes mayores de 75 años, que suelen ser excluidos en la mayoría de los trabajos sobre este tema ${ }^{4,5}$.

Lo que conocemos se extrapola de otros estudios, de algunos análisis de subgrupos y de contadas publicaciones orientadas a este grupo. Se pos- tula que la alta mortalidad que se observa en estos pacientes depende, primero de la edad, elemento reconocido como factor esencial, además de otros factores como una mayor frecuencia de presentaciones atípicas del síndrome coronario agudo, consultas más tardías, electrocardiografía menos típica, mayor comorbilidad y con una alarmante frecuencia, falta de aplicación de tratamientos probadamente útiles ${ }^{4,5,6}$. Un hecho trascendente a considerar en una buena práctica clínica, transversal a cualquier especialidad médica, es el cambio que ha experimentado en los últimos años la expectativa de vida y las características demográficas de la población tanto en Chile y en el mundo. Para el año 2025, se espera en nuestra población 20\% de mayores de 65 años $^{7}$, siendo esencial conocer experiencias locales en este grupo en particular.

Este artículo pretende revisar la experiencia 
de un centro universitario en el IAM en pacientes chilenos adultos mayores, determinar sus características clínicas, evolución y tratamiento hospitalario, mortalidad, tanto hospitalaria como al seguimiento hasta 5 años y comparar estas características con el grupo de menor edad. También se pretende evaluar los cambios temporales en la evolución y tratamiento de este grupo durante 15 años de registro. Estos dos objetivos van en busca de caracterizar a este grupo y buscar factores tanto modificables como no modificables que se asocien a las diferencias de evolución descritas en la literatura.

\section{Material y Método}

Se realiza un estudio descriptivo analítico desde un registro de todos los pacientes que ingresan primariamente por IAM a nuestra Unidad Coronaria desde enero de 1988 hasta diciembre de 2002. Para definir la presencia de IAM se utiliza la definición propuesta por la OMS, basada en sintomatología, electrocardiograma y biomarcadores $\mathrm{CK}-\mathrm{CKMB}^{8}$. Se seleccionan todos los pacientes $\geq 65$ años. Se describen sus características clínicas, su evolución hospitalaria, mortalidad y sus causas. Se realiza seguimiento de hasta 5 años en cuanto a mortalidad, a través de revisión de ficha clínica, llamado telefónico y en último término, consulta al registro civil. Se compara con el grupo de pacientes menores de 65 años. Para el análisis de las características del IAM, manejo y desenlaces de manera evolutiva, se segmentó al grupo estudiado en tres grupos de 5 años consecutivos, cada uno $(G)$ según año de ingreso (G1: 1988-1992, G2: 1993-1997, G3: 19982002) y se comparan sus características clínicas, evolución hospitalaria, tratamientos recibidos y seguimiento en cuanto a mortalidad a 5 años. Para la estadística se usó la prueba de $\chi^{2}$ para variables categóricas y $\mathrm{t}$ de Student para continuas con $\mathrm{p}<0,05$ como significativo.

\section{Resultados}

En el período observado, entre 1988 y 2002 ingresaron 1.358 pacientes con diagnóstico de IAM, de ellos 580 (42,7\%) fueron adultos mayores (PAM). Setecientos setenta y ocho pacientes tenían $<$ de 65 años (Pm). En la Tabla 1 se muestran las características generales de los dos grupos y la comparación entre ambos. Se observa predominio de sexo masculino con mayor proporción en el grupo de menor edad. Entre los factores de riesgo cardiovascular (FRCV) los PAM tenían significativamente más proporción de hipertensión arterial (HTA) y diabetes (DM), el grupo Pm tenía una proporción más elevada de tabaquismo, dislipidemia y obesidad. En cuanto a antecedentes, los PAM presentaban mayor proporción de historia de angor e infarto al miocardio previos.

En la Tabla 1 también se muestran las características clínicas de los pacientes al ingreso. Los PAM ingresaron en promedio a las $11 \mathrm{~h}$ de evolución de su IAM, en su mayoría en Killip 1 $(62 \%)$, predominando el IAM con supradesnivel ST (IAMSDST), siendo considerable el IAM de pared anterior. La mayoría desarrolló onda Q. Comparado con los Pm, el grupo de PAM tuvo mayor compromiso hemodinámico (mayor frecuencia cardiaca $(\mathrm{Fc})$, menor presión arterial sistólica (Pas) y menor proporción de Killip 1), menor proporción de IAMSDST y onda q, con una mayor incidencia significativa de IAM anterior.

La terapia inicial (Tabla 2) mostró diferencias significativas entre ambos grupos, el grupo PAM recibió menor terapia de reperfusión, a expensas de menor trombolisis, así como también recibió menos B-bloqueo (b-b), heparina y nitroglicerina (Ntg) endovenosa. No se observan diferencias en cuanto al uso de aspirina.

En cuanto a la evolución hospitalaria (Tabla 2), los PAM evolucionaron con una alta frecuencia de complicaciones (59\%), destacando la insuficiencia cardiaca (79\%). Un alto porcentaje se estudió con ecocardiografía y un significativo número recibió estudio angiográfico, pero, sólo uno de cada cuatro fue revascularizado, sea con angioplastía (Ap) o cirugía (cir). Estadía hospitalaria promedio de 14 días, con una mortalidad intrahospitalaria alta, de 143 pacientes $(24,6 \%)$, principalmente por insuficiencia cardiaca (56\%) y complicaciones mecánicas (20\%). Cuando comparamos los grupos, los PAM tuvieron significativamente menor estudio intrahospitalario: tanto con ecocardiograma como angiografía coronaria, con una menor proporción de revascularización por cualquier método $(24,8 \%$ vs $38,7 \%$, p $<$ ). Tuvieron un nivel mayor de complicaciones y mortalidad intrahospitalaria mayor $(24,6$ vs $7,6 \%, \mathrm{p}<)$.

En la Tabla 3 se presenta el tratamiento al alta y el seguimiento en cuanto a mortalidad a 5 años. Los PAM recibieron un alto porcentaje de 
Tabla 1. Características generales de los grupos y al ingreso

\begin{tabular}{|c|c|c|c|c|c|}
\hline & $\mathbf{n}$ & $\%$ & $\mathbf{n}$ & $\%$ & $\mathbf{p}$ \\
\hline Grupo & PAM: 580 & 42,7 & Pm: 778 & 57,3 & - \\
\hline Edad & $73,5 \pm 6,2 \quad(65-93)$ & & $53,0 \pm 8,3(24-64)$ & - & $<0,001$ \\
\hline Sexo masculino & 388 & 66,9 & 643 & 82,6 & $<0,001$ \\
\hline Tabaco & 154 & 26,6 & 494 & 63,5 & $<0,001$ \\
\hline HTA & 356 & 61,3 & 313 & 40,2 & $<0,001$ \\
\hline DM & 147 & 25,3 & 144 & 18,5 & $<0,001$ \\
\hline Dislipidemia & 67 & 11,6 & 153 & 19,7 & $<0,001$ \\
\hline Obesidad & 50 & 8,6 & 117 & 15,0 & $<0,001$ \\
\hline Angor previo & 283 & 48,8 & 298 & 38,3 & $<0,001$ \\
\hline IAM previo & 102 & 17,9 & 79 & 10,2 & $<0,001$ \\
\hline Revasc. previo & 25 & 4,3 & 34 & 4,8 & 0,94 \\
\hline Horas evolución & $10,8 \pm 13,9 \quad(1-72)$ & - & $9,7 \pm 12,7(1-72)$ & - & 0,13 \\
\hline $\mathrm{Fc}$ & $82,8 \pm 20,7$ (30-150) & - & $80,9 \pm 19(30-145)$ & - & 0,08 \\
\hline Pas & $127,1 \pm 27,5(50-220)$ & - & $130 \pm 26(50-220)$ & - & 0,048 \\
\hline Killip 1 & 362 & 62,4 & 653 & 83,9 & $<0,001$ \\
\hline Ck max & $1.402 \pm 2.012(170-12.889)$ & - & $1.742 \pm 1.748(207-12.239)$ & - & $<0,001$ \\
\hline Ckmb max & $133 \pm 130(23-907)$ & - & $159,2 \pm 164,6(20-1.151)$ & - & 0,002 \\
\hline Hora peak & $19,8 \pm 6,6(4-36)$ & & $15,9 \pm 6,2(4-36)$ & - & $<0,001$ \\
\hline Ascenso st & 423 & 72,9 & 606 & 77,9 & 0,04 \\
\hline Descenso st & 265 & 45,7 & 340 & 43,7 & \\
\hline Onda q & 351 & 60,5 & 525 & 67,5 & 0,009 \\
\hline lam anterior & 317 & 54,7 & 360 & 46,3 & 0,003 \\
\hline
\end{tabular}

aspirina, seguido por inhibidores de la enzima convertidora (Ieca), menos de un tercio recibió b-b. Su mortalidad a los 5 años fue de 32,9\%. Al compararlos con los $\mathrm{Pm}$, los pacientes adultos mayores recibieron menos b-b, Ieca y estatinas y su mortalidad fue significativamente más alta $(32,9$ vs $12,3 \%, \mathrm{p}<0,000)$.

Para el análisis temporal se divide el grupo total de pacientes adultos mayores en tres subgrupos de 5 años cada uno, cuyas características generales y de ingreso se detallan en la Tabla 4.

Podemos apreciar que los grupos son esencialmente comparables en la mayor parte de las variables consideradas, observándose algunas diferencias menores como menor frecuencia de tabaquismo, aumento de dislipidemia en los últimos años (G3), así como menor frecuencia de supradesnivel ST y de onda q en la misma temporalidad.

En la Tabla 5 se presenta la evolución histórica en cuanto al tratamiento inicial, estudio, tratamiento, evolución y mortalidad hospitalaria, y en la Tabla 6 el tratamiento al alta y la mortalidad al seguimiento. En relación al tratamiento inicial, se aprecia un aumento significativo de la aplicación de todos los tipos de tratamiento desde los primeros 5 años en adelante, exceptuando la trombolisis, y un progresivo y significativo aumento en el uso de angioplastía primaria (App) y b-bloqueo. En el estudio hospitalario ocurre lo mismo, observándose un mayor estudio ecocardiográfico $\mathrm{y}$ angiográfico a lo largo de los tres períodos, con un mayor uso de la revascularización coronaria. En cuanto a la evolución hospitalaria, se observa una disminución progresiva de las complicaciones, así como en la mortalidad, la cual disminuyó de 
Infarto agudo al miocardio en el adulto mayor - H. Ugalde et al

Tabla 2. Tratamiento y evolución hospitalaria

\begin{tabular}{|c|c|c|c|c|c|}
\hline & PAM: > 65a & $\%$ & Pm: < 65a & $\%$ & $\mathbf{P}$ \\
\hline Trat. médico & 414 & 71,4 & 470 & 60,4 & $<0,001$ \\
\hline Reperfusión & 166 & 28,6 & 308 & 39,6 & $<0,001$ \\
\hline Lisis & 96 & 16,5 & 189 & 24,3 & $<0,001$ \\
\hline App & 70 & 12,1 & 119 & 15,3 & 0,11 \\
\hline Aspirina & 542 & 93,4 & 739 & 95,0 & 0,27 \\
\hline Ntg & 414 & 71,4 & 599 & 77,0 & 0,02 \\
\hline$b-b$ & 140 & 24,2 & 346 & 44,5 & $<0,001$ \\
\hline Heparina & 314 & 54,1 & 489 & 62,9 & 0,001 \\
\hline leca & 41 & 7,1 & 61 & 7,8 & 0,67 \\
\hline Inotropo + & 47 & 8,1 & 42 & 5,4 & 0,06 \\
\hline Iam complic. & 342 & 59,0 & 276 & 35,5 & $<0,001$ \\
\hline Eco & 481 & 82,9 & 704 & 90,5 & $<0,001$ \\
\hline Coro & 340 & 58,6 & 676 & 86,9 & $<0,001$ \\
\hline Trat. médico & 436 & 75,2 & 475 & 61,1 & $<0,001$ \\
\hline Revascul. & 144 & 24,8 & 301 & 38,7 & $<0,001$ \\
\hline Ap & 79 & 13,6 & 172 & 22,1 & $<0,001$ \\
\hline Cir & 65 & 11,2 & 129 & 16,6 & 0,003 \\
\hline Días Hosp. & $13,9 \pm 10,7 \quad(1-80)$ & & $15,1 \pm 10,1 \quad(1-90)$ & & \\
\hline Mortalidad & 143 & 24,7 & 55 & 7,6 & 0,000 \\
\hline
\end{tabular}

Tabla 3. Tratamiento al alta y mortalidad al seguimiento

\begin{tabular}{|c|c|c|c|c|c|}
\hline & PAM > 65a & $\%$ & $\mathrm{Pm}<65 a$ & $\%$ & $\mathbf{p}$ \\
\hline Aspirina & 414 & 94,5 & 697 & 96,4 & 0,22 \\
\hline$b-b$ & 154 & 31,2 & 385 & 53,3 & $<0,001$ \\
\hline B calcio & 167 & 38,1 & 263 & 36,4 & 0,57 \\
\hline leca & 204 & 46,6 & 288 & 39,8 & 0,03 \\
\hline Estatina & 89 & 20,3 & 210 & 29,0 & 0,001 \\
\hline Seguimiento & 432 & 98,9 & 721 & 99,7 & 0,15 \\
\hline Mortalidad & 142 & 32,9 & 89 & 12,3 & $<0,000$ \\
\hline Causa CV & 99 & 69,7 & 63 & 70,8 & 0,98 \\
\hline
\end{tabular}

$32,5 \%$ en los primeros años a $18,5 \%$ en el último quinquenio, p: 0,003 (representando 56,9\% de disminución en la mortalidad en el período observado). La mortalidad en el grupo de Pm también disminuyó alcanzado 44,8\%, p: 0,03.

$\mathrm{Al}$ alta ocurre el mismo hecho, se observa un significativo aumento del uso de fármacos, especialmente, b-bloqueo, inhibidores de enzima de conversión (Ieca) y estatinas. La mortalidad a largo plazo disminuyó en forma significativa, alcanzando 64,6\% de disminución (de 41,2 a 26,6\%, p 0,016). En el grupo de Pm esta disminución alcanzó a 53,8\% (16,9 a 9,1\%, p 0,013).

Una forma gráfica de ver las diferencias en 
Tabla 4. Características generales y al ingreso de los grupos

\begin{tabular}{|c|c|c|c|c|c|c|}
\hline & G1: 88-92 & G2: 93-97 & G3: 98-02 & p 1-2 & p 2-3 & p 1-3 \\
\hline Número & 169 & 206 & 205 & - & - & - \\
\hline Edad & $73,1 \mathrm{a}$ & $73,2 a$ & $74,0 a$ & 0,87 & 0,2 & 0,16 \\
\hline Sexo masc. & $64,5 \%$ & $67 \%$ & $68,7 \%$ & 0,69 & 0,78 & 0,44 \\
\hline Tabaco & $33,7 \%$ & $30,6 \%$ & $16,6 \%$ & 0,59 & 0,001 & $<0,001$ \\
\hline HTA & $58,0 \%$ & $58,3 \%$ & $67,3 \%$ & 0,96 & 0,07 & 0,08 \\
\hline DM & $20,1 \%$ & $29,2 \%$ & $30,7 \%$ & 0,08 & 0,72 & 0,03 \\
\hline Dislipidemia & $4,7 \%$ & $8,3 \%$ & $24,9 \%$ & 0,25 & $<0,001$ & $<0,001$ \\
\hline Obesidad & $5,3 \%$ & $11,7 \%$ & $13,2 \%$ & 0,05 & 0,75 & 0,02 \\
\hline Angor previo & $55,6 \%$ & $48,1 \%$ & $43,9 \%$ & 0,18 & 0,46 & 0,03 \\
\hline lam previo & $11,8 \%$ & $14,1 \%$ & $10,7 \%$ & 0,63 & 0,38 & 0,86 \\
\hline Revasc. prev. & $0,6 \%$ & $2,4 \%$ & $5,8 \%$ & 0,32 & 0,14 & 0,01 \\
\hline Horas evolución & $12,8 \pm 16,4$ & $9,8 \pm 12,6$ & $10,2 \pm 12,8$ & 0,046 & 0,75 & 0,09 \\
\hline Fc & $84,1 \pm 22,9$ & $83,4 \pm 20,5$ & $81 \pm 19,1$ & 0,76 & 0,16 & 0,15 \\
\hline Pas & $125,7 \pm 29,7$ & $126,9 \pm 27,4$ & $128,4 \pm 25,8$ & 0,69 & 0,57 & 0,27 \\
\hline Killip 1 & $52,7 \%$ & $65,5 \%$ & $67,3 \%$ & 0,015 & 0,78 & 0,005 \\
\hline CK mb max. & $93,5 \pm 68$ & $92,9 \pm 67$ & $104,8 \pm 76,1$ & 0,93 & 0,09 & 0,14 \\
\hline Ascenso st & $81,7 \%$ & $75,7 \%$ & $62,9 \%$ & 0,21 & 0,007 & 0,000 \\
\hline Descenso st & $8,9 \%$ & $13,1 \%$ & $15,1 \%$ & 0,26 & 0,66 & 0,09 \\
\hline Onda q & $67,5 \%$ & $64,6 \%$ & $50,7 \%$ & 0,63 & 0,006 & 0,002 \\
\hline Iam anterior & $59,2 \%$ & $54,9 \%$ & $51,7 \%$ & 0,46 & 0,59 & 0,18 \\
\hline
\end{tabular}

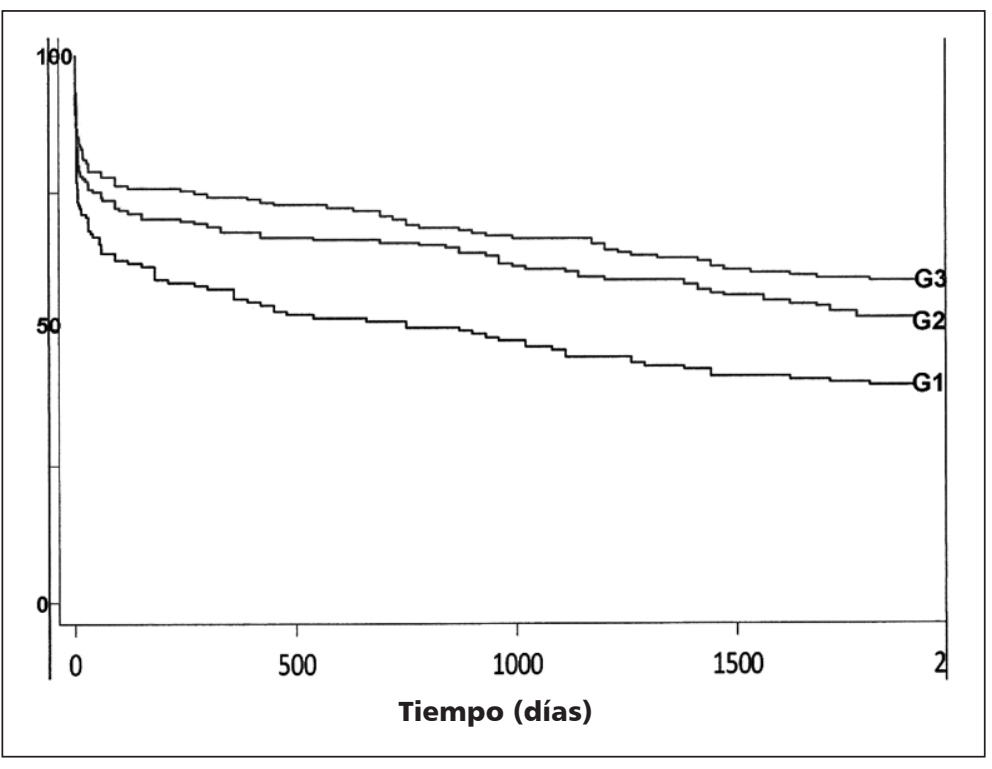

Figura 1. Curva de sobrevida según grupo-Kaplan Mier. 
Infarto agudo al miocardio en el adulto mayor - H. Ugalde et al

Tabla 5. Tratamiento inicial, estudio, tratamiento y evolución hospitalaria

\begin{tabular}{|c|c|c|c|c|c|c|}
\hline & G1: 88-92 & G2: 93-97 & G3: 98-02 & p 1-2 & p 2-3 & p 1-3 \\
\hline \multicolumn{7}{|l|}{ Trat. Inicial } \\
\hline Trat. médico & $82,2 \%$ & $68,4 \%$ & $65,8 \%$ & 0,003 & 0,28 & 0,000 \\
\hline Reperfusión & $17,8 \%$ & $31,6 \%$ & $34,1 \%$ & 0,003 & 0,28 & 0,000 \\
\hline Lisis & $17,2 \%$ & $19,0 \%$ & $13,1 \%$ & 0,76 & 0,15 & 0,35 \\
\hline App & $0,6 \%$ & $12,6 \%$ & $21,0 \%$ & 0,000 & 0,03 & 0,000 \\
\hline Aspirina & $79,3 \%$ & $99,5 \%$ & $99,0 \%$ & 0,000 & 0,99 & 0,000 \\
\hline Ntg & $54,4 \%$ & $82,0 \%$ & $74,6 \%$ & 0,000 & 0,09 & 0,000 \\
\hline b-bloqueo & $10,1 \%$ & $21,4 \%$ & $38,5 \%$ & 0,005 & 0,000 & 0,000 \\
\hline Hep & $24,9 \%$ & $61,2 \%$ & $71,7 \%$ & 0,000 & 0,03 & 0,000 \\
\hline leca & $1,2 \%$ & $7,8 \%$ & $11,2 \%$ & 0,006 & 0,3 & 0,000 \\
\hline \multicolumn{7}{|l|}{ Hospitalización } \\
\hline Eco & $62,1 \%$ & $87,4 \%$ & $95,6 \%$ & 0,000 & 0,005 & 0,000 \\
\hline Coro & $36,7 \%$ & $55,8 \%$ & $79,5 \%$ & 0,000 & 0,000 & 0,000 \\
\hline Trat. med. & $88,8 \%$ & $78,7 \%$ & $62,4 \%$ & 0,014 & 0,002 & 0,000 \\
\hline Revasc. & $11,2 \%$ & $23,3 \%$ & $37,6 \%$ & 0,014 & 0,002 & 0,000 \\
\hline Ap & $5,3 \%$ & $10,7 \%$ & $23,9 \%$ & 0,09 & 0,000 & 0,000 \\
\hline Cir & $5,9 \%$ & $12,6 \%$ & $13,7 \%$ & 0,044 & 0,87 & 0,02 \\
\hline Días hosp. & 15,9 & 14,1 & 15,3 & 0,15 & 0,2 & 0,57 \\
\hline Complicaciones & $67,5 \%$ & $56,3 \%$ & $54,6 \%$ & 0,036 & 0,8 & 0,016 \\
\hline Mortalidad PG & $32,5 \%$ & $24,3 \%$ & $18,5 \%$ & 0,097 & 0,19 & 0,003 \\
\hline Mortalidad Pm & $10,5 \%$ & $7,5 \%$ & $4,7 \%$ & 0,43 & 0,2 & 0,03 \\
\hline
\end{tabular}

Tabla 6. Tratamiento al alta y mortalidad a 5 años

\begin{tabular}{|lcccccc|}
\hline Alta-Seg. & G1: 88-92 & G2: 93-97 & G3: 98-02 & p 1-2 & p 2-3 & p 1-3 \\
\hline Aspirina & $87,7 \%$ & $95,5 \%$ & $97,6 \%$ & 0,03 & 0,47 & 0,000 \\
b-b & $20,2 \%$ & $28,2 \%$ & $51,5 \%$ & 0,17 & 0,000 & 0,000 \\
b-calcio & $47,4 \%$ & $44,2 \%$ & $21,5 \%$ & 0,7 & 0,001 & 0,000 \\
leca & $27,2 \%$ & $47,4 \%$ & $59,3 \%$ & 0,001 & 0,04 & 0,000 \\
\hline Estatina & $0,0 \%$ & $3,8 \%$ & $40,0 \%$ & 0,09 & 0,000 & 0,000 \\
Mort. 5a PG & $41,2 \%$ & $31,6 \%$ & $26,6 \%$ & 0,13 & 0,39 & 0,016 \\
\hline Mort 5a Pm & $16,9 \%$ & $13,3 \%$ & $9,1 \%$ & 0,2 & 0,27 & 0,013 \\
\hline
\end{tabular}

mortalidad entre los grupos se muestra en la Figura 1, en que se evidencia desde la hospitalización las diferencias en sobrevida y cómo se acentúan en el tiempo de seguimiento.

\section{Discusión}

El IAM en la población de pacientes mayores de 65 años es de alta incidencia, siendo un porcentaje significativo de los IAM de la población general el que se concentra en esta edad, con una mortali- dad mayor que en los pacientes menores, la cual aumenta en forma directa con la edad ${ }^{2,9}$. Pocos estudios se han publicado en el mundo referidos específicamente a la descripción y manejo realizado del IAM en este grupo, sin conocerse estudios con este objetivo en la literatura local. Recientemente se han publicado guías de recomendaciones de manejo en este grupo ${ }^{4}$, las que explícitamente reconocen utilizar información de estudios con poblaciones-objetivo distintos, y cuyas recomendaciones se basan en opinión de expertos. 
Al analizar los resultados aquí presentados, podemos afirmar la alta frecuencia que representa este grupo en el total de los IAM, con más de $40 \%$ del total de pacientes, con predominancia del sexo masculino, aunque en menor grado que en los pacientes jóvenes, confirmando lo descrito en la literatura, donde esta proporción decrece con la edad ${ }^{10}$. La HTA es el FRCV más prevalente, concordante con otros estudios ${ }^{11}$. Destaca el bajo nivel de tabaquismo, FRCV predominante en los pacientes de menor edad, esta menor incidencia puede estar dada por la existencia de algún efecto negativo del tabaco que motivó su suspensión. Existe más frecuencia de DM, hecho también asociado a la edad ${ }^{11}$. Más de la mitad de los pacientes mayores tiene antecedentes de enfermedad coronaria previa. Al ingreso, la mayoría presentó ascenso del segmento ST en el electrocardiograma inicial, hecho que según lo descrito en la literatura sería menor en este grupo de edad ${ }^{10}$. En nuestro grupo más de $70 \%$ de los pacientes mayores tenían ST elevado al ingreso, a pesar de lo cual menos de un tercio recibió alguna terapia de reperfusión, hecho concordante con la literatura, donde estos pacientes (en particular los mayores) no son considerados para estas terapias ${ }^{6,10}$ las cuales si han probado su utilidad en este rango etario ${ }^{4,12}$.

Al comparar a los pacientes mayores con el grupo de menor edad, se aprecia al ingreso mayor compromiso hemodinámico, mayor frecuencia de IAM anterior, menor terapia de reperfusión recibida y menor uso de b-bloqueo, hechos que llaman la atención, pues, claramente estos pacientes aparecen como un grupo con mayores factores asociados a mortalidad y reciben menos terapia médica y de reperfusión, hecho también consignado en otros estudios ${ }^{4,12,13}$.

En la evolución hospitalaria igualmente se aprecia una conducta más conservadora, con menos estudio angiográfico, lo que se ha asociado al mayor riesgo que potencialmente podrían tener estos estudios en los pacientes de mayor edad ${ }^{14,15}$, hecho que en experiencias locales en pacientes de edad aún mayor ( 80 y más) no se evidenció ${ }^{16}$. Al ser menos estudiados, reciben menos revascularización, a pesar de ser un grupo de mayor riesgo y que evoluciona con más complicaciones intrahospitalarias, donde una conducta activa como la revascularización tiene mejores resultados. El resultado final es una mortalidad muy superior en este grupo en comparación con el de menor edad, concordante con lo descrito en la literatura, hecho, que claramente podemos relacionar a la edad y mayor comorbilidad, pero, al cual sin duda contribuye la conducta más pasiva que se les aplica. $\mathrm{Al}$ alta, vemos que ocurre algo similar reciben menos terapia farmacológica eficaz, y su mortalidad a largo plazo es igualmente muy diferente con el grupo menor, lo cual pudiera verse explicado por los mismos argumentos anteriores. Todo esto que ocurre en este grupo es más acentuado a medida que avanza la edad ${ }^{4,10,13,15,17}$.

El punto final del estudio, y que nos conduce a proponer algunas soluciones, para los resultados ya presentados, fue el análisis de la evolución temporal, en el cual llegamos a las siguientes conclusiones, primero, los tres grupos separados por años de ingreso son similares en sus características generales y al ingreso, es decir, podemos comparar los resultados de lo que se hizo y que ocurrió, y observamos que a medida que aumenta el uso en el tratamiento inicial, tanto para la terapia de reperfusión como la terapia farmacológica asociada y también aumenta el estudio hospitalario y el tratamiento de revascularización, el resultado final es una consistente y significativa reducción de la mortalidad hospitalaria. Lo mismo ocurre al mejorar la terapia farmacológica al alta. Además de los cambios anteriormente mencionados, la mortalidad al seguimiento mejora en forma progresiva en los años de estudio. Este mismo hecho, de disminución de mortalidad, el cual ha ocurrido en todos los grupos etarios con IAM y en particular en este grupo de pacientes, concuerda con lo recientemente descrito en otros reportes, pero no se aclaran las razones por lo que esto estaría ocurriendo ${ }^{9,19,20}$, razones, que creemos serían la mejoría de la terapia farmacológica tanto inicial como al alta hospitalaria, mayor uso de terapia de reperfusión, mayor estudio intrahospitalario así como más utilización de terapias de revascularización.

Un punto distinto a lo descrito en la literatura, fue la mejoría en la mortalidad tanto hospitalaria como al seguimiento en el grupo de pacientes de más edad. Los estudios previos mostraban una mejoría mayor en los grupos de menor edad, sin claramente explicar las razones de esto ${ }^{9,18-20}$, especulándose el sub-tratamiento. Esta diferencia encontrada recalca la idea de que la mortalidad en el IAM se acumula en pacientes de más alto riesgo, como son habitualmente los de mayor edad, y que 
Infarto agudo al miocardio en el adulto mayor - H. Ugalde et al

al realizar estudios y tratamientos recomendados, especialmente en los pacientes más graves, observamos un mejor resultado clínico, como aquí se ha presentado.

\section{Conclusiones}

Este grupo de pacientes adultos mayores con IAM representa un significativo porcentaje de todos los IAM, acumulan más factores de riesgo asociados, son insuficientemente tratados y su mortalidad es muy alta, pudiendo mejorar estos resultados con una actitud terapéutica más activa, aplicando los tratamientos probadamente útiles. El impacto en la reducción de la mortalidad tanto hospitalaria como a largo plazo puede ser considerable, con un potencial beneficio respecto al grupo de menor edad. El poder iniciar este cambio radica en dejar de considerar la edad como una contraindicación para el inicio de terapias probadamente efectivas.

Limitaciones del estudio: La principal radica en que los datos presentados se inician en el año 1988 y el registro termina en 1992, y que ha habido cambios en el enfrentamiento principalmente terapéutico desde entonces, lo que ha cambiado la mortalidad, en todo caso esto no afecta la idea principal mostrada, que es que la mejoría de los tratamientos, lleva a mejores resultados, y esto es válido tanto para esa fecha como para hoy.

\section{Referencias}

1. Estadísticas de mortalidad por causas Ministerio de Salud. DEIS, MINSAL disponible en www.deis.cl consultado en abril de 2011.

2. Szot J. Mortalidad por infarto agudo al miocardio en Chile: 1990-2001. Rev Med Chile 2004; 132: 1227-33.

3. Kochanek KD, Smith BL. Deaths: preliminary data for 2002. Natl Vital Stat Rep 2004; 52 (13): 1-47.

4. Alexander K, Newby K, Armstrong P, Cannon C, Gibler B, Rich M, et al. American Heart Association Council on Clinical Cardiology: In Collaboration Infarction: A Scientific Statement for Healthcare Professionals From the Acute Coronary Care in the Elderly, Part II: ST-Segment-Elevation Myocardial With the Society of Geriatric Cardiology. Circulation 2007; 115; 2570-89.

5. Jokhadar M, Wenger N. Review of the treatment of acute coronary syndrome in elderly patients. Clinical Interventions in Aging 2009: 4; 435-44.
6. Shanmugasundaram M, Alpert J. Acute Coronary Syndrome in the Elderly. Clin Cardiol 2009; 32 (11): 608-13.

7. Marín LPP. Reflexiones para considerar en una política pública de salud para las personas mayores: Thoughts for action. Rev Med Chile 2007; 135 (3): 392-8.

8. Anonymous (March 1979). "Nomenclature and criteria for diagnosis of ischemic heart disease. Report of the Joint International Society and Federation of Cardiology/ World Health Organization task force on standardization of clinical nomenclature". Circulation 1979; 59 (3): 607-9.

9. Wellenius G, Mittleman M. Disparities in Myocardial Infarction Case Fatality Rates among the Elderly: The 20-Year Medicare Experience. Am Heart J 2008; 156 (3): 483-90.

10. Avenzum A, Makdisse M, Spencer F, Gore J, Fox K, Montalescot G, et al. Impact of age on management and outcome of acute coronary syndrome: Observations from the global registry of acute coronary events (GRACE). American Heart Journal 2005; 149 (1): 67-73.

11. Encuesta Nacional de Salud Chile 2010. En www. redsalud.gov.cl Gobierno de Chile/Ministerio de Salud. Consultado en abril de 2011.

12. Fibrinolytic Therapy Trialists Collaborative Group. Indications for fibrinolytic therapy in suspected acute myocardial infarction: collaborative overview of early mortality and major morbidity result from all randomized trials of more than 1000 patients. Lancet 1994; 343: 311-22.

13. Krumholz H, Murillo J, Chen J, Vaccarino V, Radford MJ, Ellerbeck F, et al. Thrombolytic therapy for eligible elderly patients with acute myocardial infarction. JAMA 1997; 277: 1683-8.

14. Clark VL, Khaja F. Risk of cardiac catheterization in patients aged 3-80 years without previous cardiac surgery. Am J Cardiol 1994; 74: 1076-7.

15. Moreno R, García E, Cantalapiedra J, Ortega A, López de Sa, López-Sendon J, et al. Manejo de la angina inestable: la edad avanzada continúa siendo un predictor independiente de manejo más conservador tras la estratificación pronóstica mediante prueba de esfuerzo. Rev Esp Cardiol 2000; 53: 911-8.

16. Ugalde H, Dreyse X, Espinoza P, Pizarro G. Angiografía coronaria en pacientes de 80 y más años. Experiencia de 12 años. Rev Chil Cardiol 2007; 26: 29-33.

17. Ugalde H, Espinosa P, Pizarro G, Dreyse X. Infarto agudo al miocardio en pacientes de 80 y más años. Evolución hospitalaria y seguimiento. Rev Med Chile 2008; 136: 694-700.

18. Goldberg R, McCormick D, Gurwitz J, Yarzebski J, Lessard D, Gore J. Age-related trends in short- and long- 
term survival after acute myocardial infarction: a 20-year population-based perspective (1975-1995). Am J Cardiol 1998; 82 (11): 1311-7.

19. Roger V, Jacobsen S, Weston S, Goraya T, Killian J, Reeder G, et al. Trends in the Incidence and Survival of Patients with Hospitalized Myocardial Infarction, Olmsted
County, Minnesota, 1979 to 1994. Ann Intern Med 2002; 136: 341-8.

20. Corbalán R, Nazzal C, Prieto JC, Chávez E, Lanas F, Lamich R, et al. Reducción de la mortalidad por infarto del miocardio en hospitales chilenos. Rev Med Chile 2002; 130: $368-78$ 